\title{
The Convergence of an Asynchronous Cooperative Algorithm for Distributed Power Control in Cellular Systems
}

\author{
Chi Wan Sung, Member, IEEE, and Wing Shing Wong, Senior Member, IEEE
}

\begin{abstract}
A new version of the cooperative algorithm is proposed for transmitter power control in mobile cellular systems. It is a distributed algorithm based on the assumption that limited control data communication between neighboring base stations are allowed. Its convergence under asynchronous operation is proved. Numerical results show that it has a higher convergence rate and smaller dynamic range requirement than other algorithms.
\end{abstract}

Index Terms-Asynchronous operation, distributed algorithms, power control.

\section{INTRODUCTION}

$\mathbf{P}$ OWER CONTROL is an important issue in mobile cellular systems. It is essential in determining the system capacity and the quality of service. Usually, the link quality is measured by the carrier-to-interference ratio (CIR). A question is how the transmit power of each user should be adjusted such that the CIR is optimized in some global sense.

Early work by Aein [1] has introduced the concept of CIR balancing. The idea is that all the users strive to maintain the same CIR. It is optimal in the sense that the minimum CIR of all communication links is maximized. The solution can be obtained by solving an eigenvalue problem [12]. Later, a second paradigm is adopted by Foschini and Miljanic [4]. The objective is to keep the CIR of all links above a prespecified target value. It is shown in [8] that if a feasible solution exists, there exists a unique solution which minimizes the transmit power in a Pareto sense. The solution is obtained by solving a system of linear equations.

In both of these paradigms, distributed algorithms are proposed and their convergence can be proved. However, in the CIR-balancing approach, both Zander's [13] algorithm and Grandhi et al.'s algorithm [6] need a normalization factor to scale the individual user transmit power to a desired range. The computation of the normalization factor requires global user information, thus weakening the distributed property of these algorithms. In Wong's and Lam's paper [10], a cooperative algorithm is proposed. The main idea is that some limited information flow among base stations are allowed. Of course, this kind of communication incurs a cost on the system.

Manuscript received June 3, 1996; revised December 30, 1997. This work was supported under a grant from the Hong Kong Research Grants Council.

The authors are with the Department of Information Engineering, Chinese University of Hong Kong, Shatin, N.T., Hong Kong (e-mail: cwsung3@ie.cuhk.edu.hk; wswong@ie.cuhk.edu.hk).

Publisher Item Identifier S 0018-9545(99)01032-4.
However, the requirement on global information is removed and the power control mechanism can be performed based on local measurement only.

This paper is an extension of [10], where the power of each user starts from the maximum allowed power and is adjusted monotonically downward until CIR balancing is achieved. In this work, the power of each user starts from the minimum allowed power and is adjusted monotonically upward. This modification has two advantages. First, it saves user battery life. Second, the admission of a new user minimize any sudden increase in the interference to the existing users. The disturbance to a balanced system is thus minimized.

Some related issues of the power control problem can be found in the literature. For example, the imposition of the maximum power constraint on the user transmit power was considered in [7]. The problem of user admission control was studied in [2]. In [3] and [9], power control algorithms which use a finite set of discrete levels were examined. In this paper, we focus on the asynchronous operation of the power control mechanism. This issue was first considered by Mitra [8]. He proved that Foschini's and Miljanic's algorithm [4] can be operated in an asynchronous manner. This feature relaxes the need for coordination and synchrony among base stations. Each link can have different power update rates. Moreover, the propagation delay is also taken into account. In this paper, we follow his approach and prove that the asychronous cooperative algorithm can converge and achieve optimal CIR balancing. In the last section, simulation results are presented. The issue of finite dynamic range [7], [11] is considered, and the convergence rate of different algorithms is compared.

\section{SySTEM Model}

In this section, we present the system model and some relevant results. Consider a cellular radio system. To each communication link, we allocate a pair of orthogonal channels (time slots or frequencies) for the mobile-to-base (uplink) and the base-to-mobile (downlink) communication. Since there is no interference between the uplinks and the downlinks, in this paper we consider power control for the uplinks only. However, the results can be applied for the downlinks as well.

Now, let us focus on a set of cells in which a particular channel is used in a particular instant. Let $K$ be the cardinality of this set. Let $P_{i}$ be the power transmitted by the $i$ th mobile. 
The link gain from mobile $j$ to base station $i$ at a particular instant is denoted by $G_{i j}$. The matrix $G=\left\{G_{i j}\right\}$ is known as the uplink gain matrix. In our model, we ignore the effect of adjacent channel interference and thermal noise. Thus, the CIR at base station $i, \Gamma_{i}$ can be written as

$$
\Gamma_{i}=\frac{G_{i i} P_{i}}{\sum_{j \neq i} G_{i j} P_{j}}
$$

Equation (1) can be rewritten more compactly as

$$
\Gamma_{i}=\frac{P_{i}}{\sum_{j \neq i} Z_{i j} P_{j}}
$$

where the normalized uplink gain matrix $Z$ is defined by $Z_{i j}=G_{i j} / G_{i i}$.

It should be noted that in mobile cellular systems, the link gains change constantly in time. Thus, the link gain matrix $G$ is a stochastic process. In our model, we consider a snapshot of the system such that $\boldsymbol{G}$ is treated as a $K \times K$ matrix of random variables.

Let $P=\left(P_{1}, P_{2}, \cdots, P_{K}\right)$ be the vector of the $K$ users' transmit power. A CIR $\gamma$ is defined to be achievable if there exists a power vector $\boldsymbol{P}$ with all positive components such that $\Gamma_{i} \geq \gamma$ for all $i$. The notation $\boldsymbol{P}>0$ is used to denote that all the components of $\boldsymbol{P}$ are positive. It was proved in [12] that the maximum achievable CIR $\gamma^{*}$ equals

$$
\gamma^{*}=\max _{\boldsymbol{P}>0} \min _{i} \Gamma_{i}=\frac{1}{\lambda^{*}-1}
$$

where $\lambda^{*}$ is the largest real eigenvalue of $\boldsymbol{Z}$. Moreover, an eigenvector corresponding to the eigenvalue $\lambda^{*}$ yields the balanced solution

$$
\Gamma_{i}=\gamma^{*}, \quad \forall i
$$

\section{POWER CONTROL AlgORITHM}

In our model, we made the same assumption as in [10], where the base stations are interconnected by a wired backbone network so that control data information can be sent from one base station to another. Obviously, there is a cost associated with this kind of information flow. To minimize this cost, the transmission of control data from a base station should be restricted to its network neighbors as much as possible. By network neighbors we refer to those base stations between which the data communication costs are small. This is determined by the topology of the wired backbone network.

To define the way how the power control data are passed among the base stations, we use the control data flow structure. This structure is a directed graph where each node represents a base station. If there is a directed arc from node $A$ to node $B$, then control data are passed from base station $A$ to $B$. In addition, we assume that the control data flow structure satisfies the following.
Reachability Condition: A control data flow structure satisfies the reachability condition if for any pair of nodes $(A, B)$ there is a chain of directed arcs starting from $A$ and terminating at $B$.

This is a sufficient condition for the convergence of the proposed algorithm as will be seen in Theorem 2 .

The proposed power control algorithm is a discrete-time algorithm. It is similar to the algorithm in [10]. At each iteration, each base station computes the transmit power of its serviced mobile terminal based on the previous power level, its current CIR and the CIR's it receives from its neighbors. The algorithm is stated as follows.

\section{A. The Cooperative Algorithm}

Let $\mathcal{N}_{i}$ be the set of indexes of base stations that send control data information to base station $i$ according to the control data flow structure. In the $(n+1)$ th step, each mobile unit adjusts its transmit power $P_{i}^{(n+1)}$ according to the following rule:

$$
\begin{aligned}
P_{i}^{(0)} & =P_{\min } \\
P_{i}^{(n+1)} & =\alpha_{i}^{(n)} P_{i}^{(n)} \\
\alpha_{i}^{(n)} & =\sqrt[m]{\frac{\max \left(\Gamma_{i}^{(n)}, c \max _{j \in \mathcal{N}_{i}} \Gamma_{j}^{(n)}\right)}{\Gamma_{i}^{(n)}}}
\end{aligned}
$$

where $m \geq 1$ is a parameter of the algorithm that controls the convergence rate and $c$ is a constant satisfying $1-\epsilon<c<1$ for arbitrary small $\epsilon>0$. The constant $c$ is needed for the proof of the convergence of the algorithm. The condition $c<1$ ensures that the limit of the power level, $\lim _{n \rightarrow \infty} P_{i}^{(n)}$, exists (see Proposition 4 and Theorem 1). Thus, the algorithm will converge to a fixed point. The condition $c>1-\epsilon$ for arbitrary small $\epsilon$ ensures that the fixed point achieves CIR balancing (see Theorem 2). The effect of $m$ will be discussed in the section of numerical studies.

The convergence property of the algorithm does not depend on the initial power level. Here, we arbitrarily assume that the initial power is the minimum transmit power. In practice, a setup phase is needed before the algorithm starts. A suitable initial power level should be chosen such that the initial CIR will not be too low which renders a proper power control operation impossible.

\section{ASYNCHRONOUS ADAPTATION}

In the above statement of the algorithm, we have assumed that time is slotted and the power level of all links update simultaneously. The resulting CIR of a base station is sent to its network neighbors instantaneously. This requires synchronous operation among the base stations [10]. In fact, these conditions can be relaxed. First, the power level can be changed in different time and in different rate. Second, the control data regarding the CIR can be sent in a rate different from the power updating. The third relaxation comes from the propagation delay. Consider the scenario where mobile $i$ updates its power level just after mobile $j$ 's update. At the instant when mobile $i$ updates its power, the effect of mobile $j$ 's update may not have 
propagated to it. As a result, mobile $i$ 's update is not based on the latest value of mobile $j$ 's power, but a prior value instead.

Our model incorporates the above three forms of asynchronous operation. We use $n$ as an index for subsequent power update in the whole system. Note that the time between two consecutive updates can be varied. At the $n$th step, one or more mobiles can update their power concurrently. Since the control data can be sent in a different rate and at different time, at the $n$th step, the information, $\left\{\Gamma_{j}^{(n)}: j \in \mathcal{N}_{i}\right\}$, stored in base station $i$ may not be the most recent value. We denote them as $\left\{\hat{\Gamma}_{j}^{(i, n)}: j \in \mathcal{N}_{i}\right\}$. Moreover, we assume that $\hat{\Gamma}_{j}^{(i, n)}=\Gamma_{j}^{\left(n-d_{i j}\right)}$ for some $d_{i j} \leq d_{\max }$. It means that the delay in control data transmission is bounded by $d_{\max }$.

To take into account the propagation delay, the interference from mobile $j$ to base station $i$ at step $n$ is denoted by $Z_{i j} \hat{P}_{j}^{(i, n)}$, where $\hat{P}_{j}^{(i, n)}=P_{j}^{\left(n-e_{i j}\right)}$ for some $e_{i j} \geq 0$. Accordingly, the measured CIR at base station $i$ becomes

$$
\hat{\Gamma}_{i}^{(i, n)}=\frac{P_{i}^{(n)}}{\sum_{j \neq i} Z_{i j} \hat{P}_{j}^{(i, n)}}
$$

\section{A. The Asynchronous Cooperative Algortihm}

Assume that mobile $i$ adjusts its transmit power at step $n+1$. Its power is adjusted according to the following rule:

$$
\begin{aligned}
P_{i}^{(n+1)} & =\alpha_{i}^{(n)} P_{i}^{(n)} \\
\alpha_{i}^{(n)} & =\sqrt[m]{\frac{\max \left(\hat{\Gamma}_{i}^{(i, n)}, c \max _{j \in \mathcal{N}_{i}} \hat{\Gamma}_{j}^{(i, n)}\right)}{\hat{\Gamma}_{i}^{(i, n)}} .}
\end{aligned}
$$

\section{Convergence Properties}

The convergence properties of the asynchronous cooperative algorithm is summarized in the following propositions and theorems.

First, we define

$$
\Gamma_{\max }^{(n)}=\max _{j} \Gamma_{j}^{(n)}
$$

which is the maximum CIR of all the $K$ users in the system at step $n$.

Second, we define

$$
\gamma_{i}^{(n)}=\max _{j \in \mathcal{N}_{i}} \hat{\Gamma}_{j}^{(i, n)}
$$

which is the maximum CIR of the information stored at base station $i$.

Equation (10) can then be rewritten as

$$
\alpha_{i}^{(n)}=\sqrt[m]{\frac{\max \left(\hat{\Gamma}_{i}^{(i, n)}, c \gamma_{i}^{(n)}\right)}{\hat{\Gamma}_{i}^{(i, n)}}} .
$$

Proposition 1: The power level $P_{i}^{(n)}$ is a monotone increasing function of $n$. Moreover, the function

$$
\Lambda^{(n)}=\max _{0 \leq d \leq d_{\max }} \Gamma_{\max }^{(n-d)}
$$

is a monotone decreasing function of $n$.
Proof: Since $\alpha_{i}^{(n)} \geq 1$, it follows directly that the power level $P_{i}^{(n)}$ is monotone increasing.

For mobile $i$ where $\hat{\Gamma}_{i}^{(i, n)} \geq c \gamma_{i}^{(n)}$

$$
\Gamma_{i}^{(n+1)}=\frac{P_{i}^{(n)}}{\sum_{j \neq i} Z_{i j} P_{j}^{(n+1)}} \leq \Gamma_{i}^{(n)} \leq \Gamma_{\max }^{(n)} \leq \Lambda^{(n)} .
$$

For mobile $i$ where $\hat{\Gamma}_{i}^{(i, n)}<c \gamma_{i}^{(n)}$

$$
\begin{aligned}
\Gamma_{i}^{(n+1)} & =\left(\frac{c \gamma_{i}^{(n)}}{\hat{\Gamma}_{i}^{(i, n)}}\right)^{1 / m} \frac{P_{i}^{(n)}}{\sum_{j \neq i} Z_{i j} P_{j}^{(n+1)}} \\
& \leq \frac{c \gamma_{i}^{(n)} P_{i}^{(n)}}{\hat{\Gamma}_{i}^{(i, n)} \sum_{j \neq i} Z_{i j} P_{j}^{(n+1)}} \\
& =\frac{c \gamma_{i}^{(n)} \sum_{j \neq i} Z_{i j} \hat{P}_{j}^{(i, n)}}{\sum_{j \neq i} Z_{i j} P_{j}^{(n+1)}} \\
& \leq \frac{c \gamma_{i}^{(n)} \sum_{j \neq i} Z_{i j} P_{j}^{(n)}}{\sum_{j \neq i} Z_{i j} P_{j}^{(n+1)}} \\
\leq c \gamma_{i}^{(n)} & \leq c \max _{0 \leq d \leq d_{\max }} \Gamma_{\max }^{(n-d)} \\
\leq \Lambda &
\end{aligned}
$$

Combining (14) and (15), we have

$$
\Gamma_{i}^{(n+1)} \leq \Lambda^{(n)}
$$

for all $i$.

In particular

$$
\Gamma_{\max }^{(n+1)} \leq \Lambda^{(n)}
$$

which implies

$$
\begin{aligned}
\Lambda^{(n+1)} & =\max _{0 \leq d \leq d_{\max }} \Gamma_{\max }^{(n+1-d)} \\
& \leq \max _{0 \leq d \leq d_{\max }+1} \Gamma_{\max }^{(n+1-d)} \\
& =\max \left(\Lambda^{(n)}, \Gamma_{\max }^{(n+1)}\right) \\
& =\Lambda^{(n)} .
\end{aligned}
$$

Proposition 2: For all $n$

$$
\Lambda^{(n)} \geq \gamma^{*}
$$

where $\gamma^{*}$ is the maximum achievable CIR.

Moreover, the limit

$$
\Lambda^{*}=\lim _{n \rightarrow \infty} \Lambda^{(n)}
$$

exists and $\Lambda^{*} \geq \gamma^{*}>0$. 
Proof: By a corollary of the Frobenius Theorem [5, p. 65], it follows that for any power control assignment $\boldsymbol{P}$ with all positive components, the CIR satisfies the condition

$$
\min _{i} \Gamma_{i}(P) \leq \gamma^{*} \leq \max _{i} \Gamma_{i}(P)
$$

where $\gamma^{*}$ is the maximum achievable CIR. The equality holds if and only if

$$
\Gamma_{i}(\boldsymbol{P})=\gamma^{*}, \quad \text { for all } i
$$

Since $\gamma^{*}$ is a lower bound of $\Gamma_{\max }^{(n)}$ for all $n$, it is also a lower bound of $\Lambda^{(n)}$ for all $n$. The second statement then follows from the fact that $\Lambda^{(n)}$ is monotone decreasing with $\gamma^{*}$ as a lower bound.

Proposition 3: If $\hat{\Gamma}_{i}^{(i, N)} \leq c \Lambda^{(N)}$, then $\hat{\Gamma}_{i}^{(i, N+k)} \leq c \Lambda^{(N)}$ for all $k \geq 0$.

Proof: It is obvious that the proposition is true for $k=0$. Assume that it is true for $k=l$. Now we need to prove that $\hat{\Gamma}_{i}^{(i, N+l)} \leq c \Lambda^{(N)}$ implies $\hat{\Gamma}_{i}^{(i, N+l+1)} \leq c \Lambda^{(N)}$.

First, we consider the case

$$
\hat{\Gamma}_{i}^{(i, N+l)}<c \Lambda^{(N+l)} .
$$

Note that, for all $n$

$$
\gamma_{i}^{(n)} \leq \max _{j} \hat{\Gamma}_{j}^{(i, n)}=\max _{j} \Gamma_{j}^{\left(n-d_{i j}\right)} \leq \Lambda^{(n)} .
$$

Then the multiplicative factor of the power control algorithm $\alpha_{i}^{(N+l)}$ satisfies the following inequality:

$$
\begin{aligned}
\alpha_{i}^{(N+l)} & =\sqrt[m]{\frac{\max \left(\hat{\Gamma}_{i}^{(i, N+l)}, c \gamma_{i}^{(N+l)}\right)}{\hat{\Gamma}_{i}^{(i, N+l)}}} \\
& \leq \sqrt[m]{\frac{c \Lambda^{(N+l)}}{\hat{\Gamma}_{i}^{(i, N+l)}}} \\
& \leq \frac{c \Lambda^{(N+l)}}{\hat{\Gamma}_{i}^{(i, N+l)}}
\end{aligned}
$$

where the first inequality follows from (19) and (20).

As a result

$$
\begin{aligned}
\hat{\Gamma}_{i}^{(i, N+l+1)} & =\frac{\alpha_{i}^{(N+l)} P_{i}^{(N+l)}}{\sum_{j \neq i} Z_{i j} \hat{P}_{j}^{(i, N+l+1)}} \\
& \leq \frac{c \Lambda^{(N+l)} P_{i}^{(N+l)}}{\hat{\Gamma}_{i}^{(i, N+l)} \sum_{j \neq i} Z_{i j} \hat{P}_{j}^{(i, N+l+1)}} \\
& =\frac{c \Lambda^{(N+l)} \sum_{j \neq i} Z_{i j} \hat{P}_{j}^{(i, N+l)}}{\sum_{j \neq i} Z_{i j} \hat{P}_{j}^{(i, N+l+1)}} \\
& \leq c \Lambda^{(N+l)} \\
& \leq c \Lambda^{(N)}
\end{aligned}
$$

where the last two inequalites follows from Proposition 1.
Next, we consider the case

$$
\hat{\Gamma}_{i}^{(i, N+l)} \geq c \Lambda^{(N+l)} .
$$

According to the power control algorithm, we have $\alpha_{i}^{(N+l)}=1$, which implies

$$
P_{i}^{(N+l+1)}=P_{i}^{(N+l)} .
$$

Therefore

$$
\hat{\Gamma}_{i}^{(i, N+l+1)} \leq \hat{\Gamma}_{i}^{(i, N+l)} \leq c \Lambda^{(N)}
$$

where the first inequality follows from the monotonicity of $\hat{P}_{j}$ and the second one from the induction hypothesis.

By induction, the proposition is true for all $k \geq 0$.

Proposition 4: There exists $i$ and $N$ such that $c \Lambda^{(n)} \leq$ $\hat{\Gamma}_{i}^{(i, n)} \leq \Lambda^{(n)}$ for all $n \geq N$.

Proof: First, we assume that the proposition does not hold.

Arbitrarily choose $n_{1} \geq 0$. For mobile $i$, where $\hat{\Gamma}_{i}^{\left(i, n_{1}\right)}<c \Lambda^{\left(n_{1}\right)}$, by Proposition 3

$$
\hat{\Gamma}_{i}^{\left(i, n_{1}+k\right)} \leq c \Lambda^{\left(n_{1}\right)}
$$

for all $k \geq 0$.

For any mobile $j$ where $\hat{\Gamma}_{j}^{\left(j, n_{1}\right)} \geq c \Lambda^{\left(n_{1}\right)}$, by the assumption, there exists $s_{j}>n_{1}$ such that

$$
\hat{\Gamma}_{j}^{\left(j, s_{j}\right)}<c \Lambda^{\left(s_{j}\right)} .
$$

Again, by proposition 3

$$
\hat{\Gamma}_{j}^{\left(j, s_{j}+k\right)} \leq c \Lambda^{\left(s_{j}\right)} \leq c \Lambda^{\left(n_{1}\right)}
$$

for all $k \geq 0$.

As a result, there exists $s=\max _{j} s_{j}>n_{1}$ such that

$$
\max _{i} \hat{\Gamma}_{i}^{(i, n)} \leq c \Lambda^{\left(n_{1}\right)}
$$

for all $n \geq s$.

Notice that

$$
\begin{aligned}
\hat{\Gamma}_{i}^{(i, n)} & =\frac{P_{i}^{(n)}}{\sum_{j \neq i} Z_{i j} \hat{P}_{j}^{(i, n)}} \\
& \geq \frac{P_{i}^{(n)}}{\sum_{j \neq i} Z_{i j} P_{j}^{(n)}} \\
& =\Gamma_{i}^{(n)} .
\end{aligned}
$$

Equations (29) and (30) imply that

$$
\Gamma_{\max }^{(n)} \leq c \Lambda^{\left(n_{1}\right)}
$$

for all $n \geq s$.

Therefore, there exists $n_{2} \geq s+d_{\max }>n_{1}+d_{\max }$ such that

$$
\Lambda^{\left(n_{2}\right)}=\max _{0 \leq d \leq d_{\max }} \Gamma_{\max }^{\left(n_{2}-d\right)} \leq c \Lambda^{\left(n_{1}\right)} .
$$

Repeating the same argument, there exists a strictly increasing sequence $\left\{n_{i}\right\}$ such that

$$
\Lambda^{\left(n_{i+1}\right)} \leq c \Lambda^{\left(n_{i}\right)} .
$$


Since $\Lambda^{(n)}$ is a monotone decreasing sequence and $c<1$, it implies that $\Lambda^{*}=0$. This contradicts the fact that $\Lambda^{*} \geq \gamma^{*}>0$ (Proposition 2).

Theorem 1: The limit of the power level $P_{i}^{*}=$ $\lim _{n \rightarrow \infty} P_{i}^{(n)}$ exists for all $i$.

Proof: By Proposition 4, there exists $i$ such that $c \Lambda^{(n)} \leq$ $\hat{\Gamma}_{i}^{(i, n)} \leq \Lambda^{(n)}$ for all $n \geq N$. According to the control algorithm, $P_{i}^{(n)}$ is a constant for all $n \geq N$. Denote it by $P_{i}^{*}$.

We can find an upper bound on $P_{j}^{(n)}(j \neq i$ and $n \geq N)$ by the following:

$$
\begin{aligned}
\hat{\Gamma}_{i}^{\left(i, n+e_{i j}\right)} & \leq \frac{P_{i}^{\left(n+e_{i j}\right)}}{Z_{i j} \hat{P}_{j}^{\left(i, n+e_{i j}\right)}} \\
& =\frac{P_{i}^{\left(n+e_{i j}\right)}}{Z_{i j} P_{j}^{(n)}} \\
P_{j}^{(n)} & \leq \frac{P_{i}^{\left(n+e_{i j}\right)}}{Z_{i j} \hat{\Gamma}_{i}^{\left(i, n+e_{i j}\right)}} \\
& \leq \frac{P_{i}^{*}}{Z_{i j} c \Lambda^{\left(n+e_{i j}\right)}} \\
& \leq \frac{P_{i}^{*}}{Z_{i j} c \gamma^{*}}
\end{aligned}
$$

where the last inequality follows from Proposition 2.

By Proposition $1, P_{j}^{(n)}$ is a monotone increasing sequence. Since $P_{j}^{(n)}$ is bounded from above, the limit

$$
P_{j}^{*}=\lim _{n \rightarrow \infty} P_{j}^{(n)}
$$

exists for all $j$.

Theorem 2: The limit of the CIR, $\lim _{n \rightarrow \infty} \Gamma_{i}^{(n)}$, exists and is arbitrarily close to $\gamma^{*}$ for all $i$.

Proof: By Theorem 1, the limit of $P_{i}^{(n)}$ exists. Denote it by $P_{i}^{*}$. Since $P_{i}^{*}>0$ for all $i$, the limit of CIR exists also. Denote it by $\Gamma_{i}^{*}$. Since we have assumed that the delay in control data transmission is bounded, i.e.,

$$
\hat{\Gamma}_{j}^{(i, n)}=\Gamma_{j}^{\left(n-d_{i j}\right)}
$$

for some $d_{i j} \leq d_{\max }$, the limit of $\hat{\Gamma}_{j}^{(i, n)}$ also exists and

$$
\lim _{n \rightarrow \infty} \hat{\Gamma}_{j}^{(i, n)}=\Gamma_{j}^{*}
$$

for all $i$ and $j$.

Consequently, the limit of $\alpha_{i}^{(n)}$ exists and we denote it by $\alpha_{i}^{*}$.

From the cooperative algorithm

$$
P_{i}^{*}=\alpha_{i}^{*} P_{i}^{*}
$$

which implies that $\alpha_{i}^{*}=1$. This in turn implies that [by (10)]

$$
\Gamma_{i}^{*} \geq c \max _{j \in \mathcal{N}_{i}} \Gamma_{j}^{*}
$$

for all $i$.

Let $\Gamma_{i}^{*}=\Gamma_{\max }^{*}$. By the reachability assumption, for any node $j$, there is a chain of directed arcs starting from node $i$ to node $j$. Let the length of the chain be $m$ and label the nodes

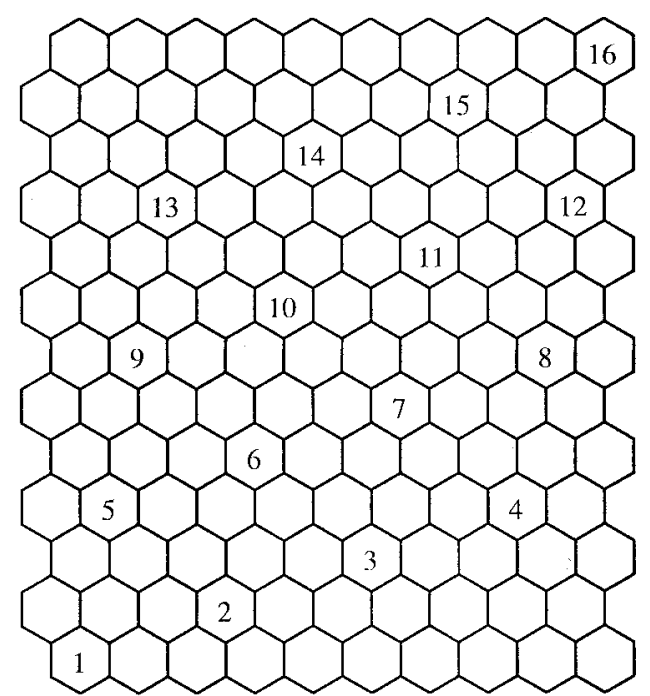

Fig. 1. Layout of interfering cells in the numerical study.

in the chain as $k_{0}=i, k_{1}, k_{2}, \cdots, k_{m}=j$. Since $k_{0} \in \mathcal{N}_{k_{1}}$, it follows that

$$
\Gamma_{k_{1}}^{*} \geq c \Gamma_{k_{0}}^{*} .
$$

By the same argument, we obtain

$$
\Gamma_{j}^{*}=\Gamma_{k_{m}}^{*} \geq c \Gamma_{k_{m-1}}^{*} \geq \cdots \geq c^{m} \Gamma_{k_{0}}^{*}=c^{m} \Gamma_{i}^{*} .
$$

Therefore, for any $j$

$$
\Gamma_{j}^{*} \geq c^{K-1} \Gamma_{\max }^{*}
$$

where $K$ is the number of base stations.

Since $c$ is arbitrarily close to one, $\Gamma_{j}^{*}$ is arbitrarily close to $\Gamma_{\max }^{*}$ for all $j$. By the corollary of Frobenius Theorem, $\Gamma_{i}^{*}$ is arbitrarily close to $\gamma^{*}$ for all $i$.

\section{NUMERICAL STUdies}

We have performed some simulation studies on the cooperative algorithm. For simplicity, the synchronous version is used. Our simulation model is the same as that in [10]. We assume a standard hexagonal cellular layout with 16 cochannel cells (see Fig. 1). The geographical location of the cells corresponds to a reuse pattern of seven. We approximate each hexagonal cell by a circular cell of the same area. Within each cell, there is a mobile terminal communicating with the base station. The location of each mobile terminal is generated uniformly inside the cell. The link gain $G_{i j}$ is defined as

$$
G_{i j}=\frac{A_{i j}}{d_{i j}^{4}}
$$

where $d_{i j}$ is the distance between the $i$ th base station and the $j$ th mobile and $A_{i j}$ is the attenuation factor. In this study, we consider Zonly lognormal fading. Hence, we assume $A_{i j}$ is lognormal distributed with mean $0 \mathrm{~dB}$ and standard deviation $6 \mathrm{~dB}$ for all $i$ and $j$. Each component of the initial power vector is generated uniformly between 0.001 and 1. Receiver noise is assumed negligible and is not modeled.

For the cooperative algorithm, two control data flow structures are considered. Structure $S_{1}$ is a single path joining 


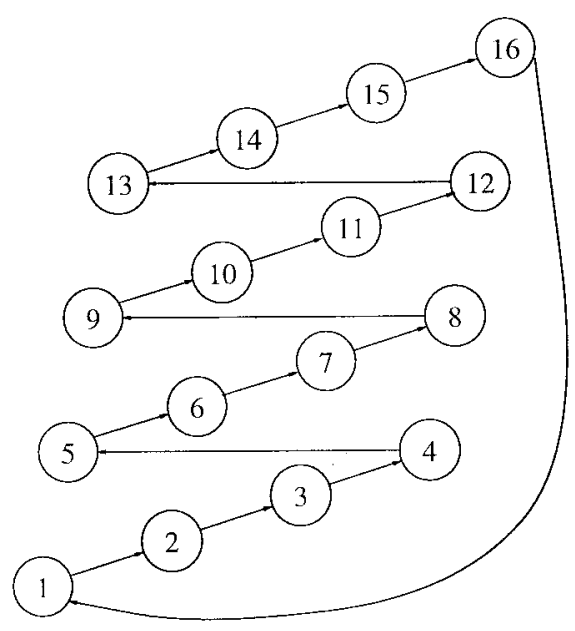

Fig. 2. Control data flow structure: $S_{1}$.

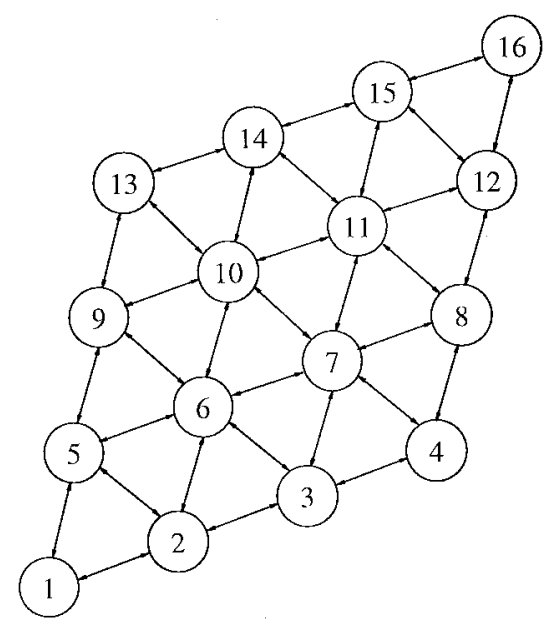

Fig. 3. Control data flow structure: $S_{2}$.

TABLE I

EFFeCT OF $m$ ON THE CONVERGENCE RATE

\begin{tabular}{c|c|c|c|c|c|c}
\hline values of $m$ & 1 & 1.1 & 1.2 & 1.3 & 1.4 & 2 \\
\hline no. of iterations for $S_{1}$ & 85.48 & 77.50 & 86.74 & 97.45 & 105.43 & 156.58 \\
\hline no. of iterations for $S_{2}$ & 84.86 & 27.52 & 27.08 & 30.12 & 33.48 & 50.32 \\
\hline
\end{tabular}

node 1 , node 2 , and up to node 16 in the same order. Node 16 is joined to node 1 in order to satisfy the reachability condition. In structure $S_{2}$, each base station sends the control data to its geographical neighbors. The two structures are shown in Figs. 2 and 3, respectively.

First, we investigate the effect of $m$ on the convergence rate. For different values of $m$, the average number of iterations needed such that the minimum CIR reaches $98 \%$ of the maximum CIR in the system is shown in Table I. The data are obtained by 1000 simulation runs. It can be seen that $m$ equals 1.1 and 1.2 is optimal for structure $S_{1}$ and $S_{2}$, respectively. We use these values for our subsequent simulations.

Next, we compare the cooperative algorithm with Zander's algorithm [12] and Grandhi et al.'s algorithm [6]. Another version of the cooperative algorithm proposed by Wong and Lam [10], in which the power levels decrease monotonically, is also shown in Table II. We call that algorithm version 1 and the newly proposed algorithm version 2 .

\section{Zander's algorithm}

$$
P_{i}^{(n+1)}=c^{(n)}\left(P_{i}^{(n)}+\frac{P_{i}^{(n)}}{\gamma_{i}^{(n)}}\right) .
$$

Grandhi's algorithm

$$
P_{i}^{(n+1)}=c^{(n)} \frac{P_{i}^{(n)}}{\gamma_{i}^{(n)}} .
$$

\section{Cooperative algorithm (version 1)}

$$
P_{i}^{(n+1)}=\alpha_{i}^{(n)} P_{i}^{(n)}
$$

where

$$
\alpha_{i}^{(n)}=\sqrt[m]{\frac{\min \left(\Gamma_{i}^{(n)}, \max \left(\min _{j \in \mathcal{N}_{i}} \Gamma_{j}^{(n)}, \gamma_{0}\right)\right)}{\Gamma_{i}^{(n)}}} .
$$

In Table II, the average number of iterations needed such that the minimum CIR reaches $85 \%, 95 \%$, and $98 \%$ of the maximum CIR is shown. It can be seen that the structure $S_{2}$ offers a higher convergence rate than $S_{1}$ at the expense of a higher communication cost. The two versions of the cooperative algorithm and Grandhi's algorithm are comparable. Zander's algorithm converges much slower than the other three.

Next, we compare the dynamic range of the power level required for these algorithms. Dynamic range is defined as the ratio between the maximum and minimum user transmit power. It is usually expressed in decibels. For Zander's and Grandhi's algorithm, we let $c^{(n)}=1$ for all $n$. A better choice for $c^{(n)}$ is suggested in [6] and [12]. However, it requires a global computation which destroys the distributed property. For the sake of a fair comparison, we simply let it be one. The initial power level is normalized to one. We stop the algorithms when the minimum CIR reaches $95 \%$ of the maximum CIR. For each case, 1000 simulation runs are performed. We obtain the minimum dynamic range such that the optimal power vector falls within it 900 times out of 1000 simulation runs. The results are shown in Table III. Grandhi's algorithm requires an extremely large dynamic range. It shows that the algorithm is not stable if the normalization constant is not set properly. For the other three algorithms, the difference on dynamic range requirement is not very large. The cooperative algorirthms are slightly better than Zander's algorithm. Considering the convergence rate and the dynamic range requirement together, the cooperative algorithm, both versions 1 and 2, compare favorably to the other two candidates.

\section{CONCLUDING REMARKS}

The cooperative algorithm was first proposed in [10]. In the original algorithm, the power level starts from the maximum value and decreases monotonically. In this paper, we propose the dual version of it. The initial power level is set as low as possible and increases monotonically when necessary. It has the advantage of saving user battery life. Besides, when a user 
TABLE II

Convergence Rate of Different Algorithms

\begin{tabular}{c|c|c|c|c|c}
\hline & Zander's & Grandhi's & $\begin{array}{c}\text { Coop. Alg. (ver.1) } \\
S_{2}, m=1.1\end{array}$ & $\begin{array}{c}\text { Coop. Alg. (ver.2) } \\
S_{1}, m=1.1\end{array}$ & $\begin{array}{c}\text { Coop. Alg. (ver.2) } \\
S_{2}, m=1.2\end{array}$ \\
\hline within $85 \%$ & 816.90 & 20.85 & 17.65 & 47.36 & 16.09 \\
\hline within $95 \%$ & 957.55 & 26.23 & 19.63 & 61.65 & 21.86 \\
\hline within $98 \%$ & 1085.52 & 30.01 & 23.51 & 77.50 & 27.08 \\
\hline
\end{tabular}

TABLE III

Dynamic Range Requirement of DifFerent Algorithms

\begin{tabular}{c|c|c|c|c}
\hline $\begin{array}{c}\text { Minimum Dynamic Rangc } \\
\text { Requirement }\end{array}$ & Zander's & Grandhi's & $\begin{array}{c}\text { Coop. Alg. (ver.1) } \\
S_{2}, m=1.1\end{array}$ & $\begin{array}{c}\text { Coop. Alg. (ver.2) } \\
S_{2}, m=1.2\end{array}$ \\
\hline $58.3 \mathrm{~dB}$ & $75.5 \mathrm{~dB}$ & $168.9 \mathrm{~dB}$ & $67.5 \mathrm{~dB}$ & $73.5 \mathrm{~dB}$ \\
\hline
\end{tabular}

TABLE IV

Typical Expected Number of Iterations for Approximately Constant Link Grain Matrix $Z$. $f=900 \mathrm{MHz}$

\begin{tabular}{c|c|c|c|c}
\hline & $\begin{array}{c}\text { Wobile Speed } \\
(\mathrm{m} / \mathrm{s})\end{array}$ & $\begin{array}{c}\text { Coherence Time of } \\
\text { Stadow Fading (s) }\end{array}$ & $\begin{array}{c}\text { Averaging Time to } \\
\text { obtain Mean CIR (s) }\end{array}$ & $\begin{array}{c}\text { Number of } \\
\text { Iterations }\end{array}$ \\
\hline small cell urban systcm & 1 & 200 & 6 & 30 \\
& 20 & 10 & 0.3 & 30 \\
\hline microccll urban system & 1 & 50 & 6 & 8 \\
& 20 & 2.5 & 0.3 & 8 \\
\hline
\end{tabular}

joins an already balanced system, the interference caused to other users at the admission instant is minimized.

The convergence of the cooperative algorithm under asynchronous operation is proved. We have also proved that the power levels always converge to finite values. However, in our analysis, we have assumed the link gain matrix $Z$ remains constant during the power control mechanism. In other words, the rate of convergence must be faster than the rate of change of the link gains due to shadow fading. Table IV shows the number of iterations needed in two different environments [13]. Comparing with the number of iterations shown in Table II, it can be seen that the cooperative algorithm would work well under traditional cellular systems with moderate cell size. For microcells or an indoor environment where the propagation changes rapidly, the averaging period in estimating CIR may need to be shortened. However, the accuracy of the CIR measurement will decrease. The impact of CIR estimation error on the algorithm may require a more detailed study.

In evaluating the performance of the cooperative algorithm, we have considered the case where there are a fixed number of users in the system. Now assume that the system has already reached the balanced state. At that time, if a new user joins the system, then a new transience will occur and eventually lead to a new balanced solution. On the other hand, if a user departs from the system, the balanced state will be disturbed. According to the algorithm, the power of the users will increase to achieve another balanced solution. However, in practice, it may not be necessary to do so. More often, we require that the CIR be greater than a certain threshold, $\gamma_{p}$, which is commonly called protection ratio. Assume that the system has reached a state where the CIR of all users are greater than $\gamma_{p}$. When a user departs, the CIR of the remaining users will only increase and thus still have a CIR greater than $\gamma_{p}$. So it is not necessary to increase the power further. In a practical situation, the cooperative algorithm can be modified as follows:

$$
\begin{aligned}
P_{i}^{(n+1)} & =\alpha_{i}^{(n)} P_{i}^{(n)} \\
\alpha_{i}^{(n)} & = \begin{cases}\sqrt[m]{\frac{\max \left(\Gamma_{i}^{(n)}, c \max _{j \in \mathcal{N}_{i}} \Gamma_{j}^{(n)}\right)}{\Gamma_{i}^{(n)}},} & \text { if } \Gamma_{i}^{(n)} \leq \gamma_{0} \\
\sqrt[m^{\prime}]{\frac{\gamma_{0}}{\Gamma_{i}^{(n)}}}, & \text { otherwise. }\end{cases}
\end{aligned}
$$

where $m^{\prime}>1$ is another control parameter. The decision threshold $\gamma_{0}$ should be greater than or equal to the protection ratio, $\gamma_{p}$.

With the above modification, the transmit power of a mobile will decrease if its received CIR at the base station is higher than $\gamma_{0}$. Therefore, in a dynamic situation where users enter and leave the system, the power level of the users in the system will not go to infinity. To verify our claim, we carried out the following simulation.

We assume the same 16-cell system as before. The CIR and the transmit power of the user in cell 6 is recorded. We assume that a departure and an arrival occurs alternately every 50 iterations. A departure is chosen randomly among the cells other than cell 6 . Fifty iterations later, an arrival occurs in the 

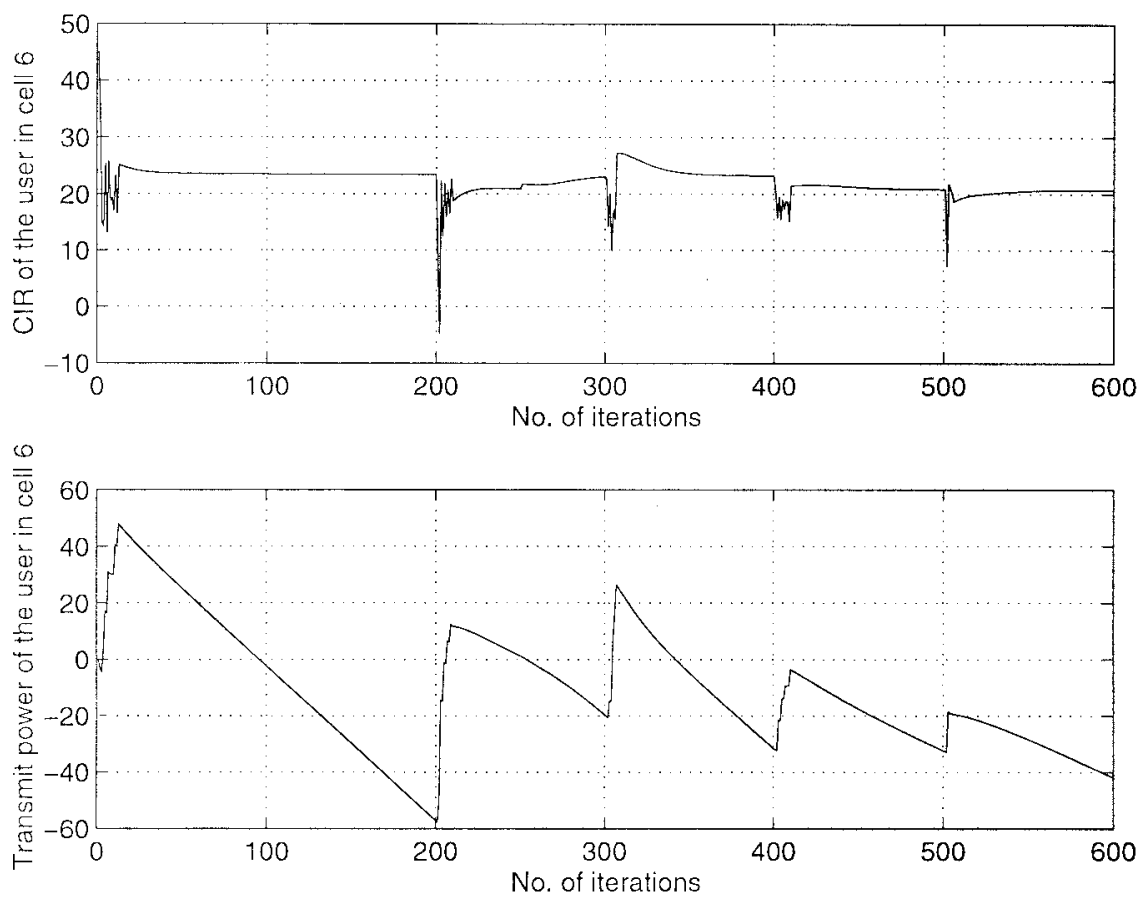

Fig. 4. The CIR and the transmit power of the user in cell 6.

previous chosen cell. The parameters of the algorithm are set as follows: $m=1.2, m^{\prime}=10$, and $\gamma_{0}=18 \mathrm{~dB}$. Note that $m^{\prime}$ is set to a relatively large value such that the decrement of the power level is more gradual. A typical simulation result is shown in Fig. 4.

It can be seen that at those arrival instants (multiples of 100), the transmit power reacts rapidly due to the sudden increase in the interference. The CIR can be brought back to an acceptable level after a short period of disturbance. When the CIR is greater than $\gamma_{0}$, the power level is adjusted downwards gradually. Therefore, in the long run, the power level can still be kept in a finite range.

This modified algorithm can still achieve CIR balancing if the maximum achievable CIR $\gamma^{*}$ is less than or equal to the threshold $\gamma_{0}$. If $\gamma^{*}$ is greater than $\gamma_{0}$, the system will reach a state such that the CIR of all mobiles is greater than $\gamma_{0}$.

\section{REFERENCES}

[1] J. M. Aein, "Power balancing in systems employing frequency reuse," COMSAT Tech. Rev., pp. 277-299, 1973.

[2] M. Andersin, Z. Rosberg, and J. Zander, "Soft admission in cellular PCS with constrained power control and noise," in Proc. 5th WINLAB Workshop, Apr. 1995.

[3] _ "Distributed discrete power control in cellular PCS," in MultiAccess, Mobility, and Teletraffic for Personal Communication. Norwell, MA: Kluwer, 1996, pp. 1-14.

[4] G. J. Foschini and Z. Miljanic, "A simple distributed autonomous power control algorithm and its convergence," IEEE Trans. Veh. Technol., vol. 42, pp. 641-646, Nov. 1993.
[5] F. R. Gantmacher, The Theory of Matrices, vol. 2. New York: Chelsea, 1959.

[6] S. A. Grandhi, R. Vijayan, and D. J. Goodman, "Distributed power control in cellular radio systems," IEEE Trans. Commun., vol. 42, pp. 226-228, Feb./Mar./Apr. 1994.

[7] S. A. Grandhi and J. Zander, "Constrained power control in cellular radio systems," in IEEE Proc. VTC '94, pp. 824-828.

[8] D. Mitra, "An asynchronous distributed algorithm for power control in cellular radio systems," in Proc. 4th WINLAB Workshop on Third Gen. Wireless Inform. Net., 1993, pp. 249-259.

[9] C. W. Sung and W. S. Wong, "A distributed fixed-step power control algorithm with quantization and active link quality protection," to be published.

[10] W. S. Wong and K. H. Lam "Distributed power balancing with a sparse information link," in IEEE Proc. VTC '94, pp. 829-832.

[11] R. D. Yates, "A framework for uplink power control in cellular radio systems," IEEE J. Select. Areas Commun., vol. 13, pp. 1341-1348, Sept. 1995.

[12] J. Zander, "Performance of optimum transmitter power control in cellular radio systems," IEEE Trans. Veh. Technol., vol. 41, pp. 57-62, Feb. 1992.

[13] _ "Distributed cochannel interference control in cellular radio systems," IEEE Trans. Veh. Technol., vol. 41, pp. 305-311, Aug. 1992.

Chi Wan Sung (M'99), for a photograph and biography, see this issue, p. 562.

Wing Shing Wong (M'81-SM'90), for a photograph and biography, see this issue, p. 562. 